\title{
Glyphosate detection by voltammetric techniques. A comparison between statistical methods and an artificial neural network
}

\author{
Nicolás Laguarda-Miro ${ }^{1,2, *}$, Francesca Werner Ferreira ${ }^{3}$, Eduardo García-Breijo ${ }^{1,4}$, Javier \\ Ibáñez-Civera ${ }^{1,4}$, Luis Gil-Sánchez ${ }^{1,4}$, José Garrigues-Baixauli ${ }^{1,4}$ \\ ${ }^{1}$ Instituto Reconocimiento Molecular y Desarrollo Tecnológico. Universitat Politècnica de València, Cami \\ de Vera s/n, 46022, Valencia, Spain. \\ 2 Departamento de Ingeniería Química y Nuclear. Universitat Politècnica de València, Cami de Vera s/n, 46022, \\ Valencia, Spain. \\ ${ }^{3}$ Universidade Regional do Noroeste do Estado do Rio Grande do Sul (UNIJUI), Rua do Comércio 3000, \\ ljuí (RS), Brazil. \\ ${ }^{4}$ Departamento de Ingeniería Electrónica. Universitat Politècnica de València, Cami de Vera s/n, 46022, \\ Valencia, Spain.
}

*Corresponding Author: nilami@iqn.upv.es

\begin{abstract}
Glyphosate quantification methods are complex and expensive, and its control in natural water bodies is getting more important year after year. In order to find a new system that facilitates the detection of glyphosate, we present a comparison between two models to predict Glyphosate concentration in aqueous dissolutions. One of them is done by an Artificial Neural Network (ANN) embedded in a Microcontroller and the other one is done by statistic methods (Partial Least Squares) in a computer.

From an analytical point of view, Voltammetric techniques have been selected to obtain electrochemical responses to different Glyphosate concentrations. In order to get them, a Voltammetry/Amperometry device designed by our research group and called FraPlus has been used. In this work we have selected two sensitive electrodes (Cobalt and Copper), ten different glyphosate concentrations and a train pulse made by nine different voltammetric pulses to build the models. The ANN developed model is able to properly relate data obtained by FraPlus and Glyphosate concentrations and the obtained value for regression coefficient $(R)$ is 0.9998 and the PValue is 0.0. Taking into account these results, we propose this ANN model based in Voltammetric techniques working with Glyphosate concentrations in a buffer as an approach to Glyphosate detection in natural water bodies.
\end{abstract}

Keywords: Artificial Neural Network, microcontroller, Glyphosate, Voltammetry, Partial Least Squares, mathematical modeling.

1. Introduction

Glyphosate is a systemic, non-selective, broad spectrum and post emergence herbicide widely used in agriculture [1]. It is chemically named as Phosphonomethyl amino acetic acid or $\mathrm{N}$ (phosphonomethyl) glycine (CAS RN 1071-83-6). This chemical is polar, non-volatile, very soluble in water and almost insoluble in organic solvents. The way this product acts as an herbicide is by inhibiting the enzyme 5-enolpyruvylshikimate-3-phosphate synthase, also known with the acronym EPSP, that has an essential function in the metabolism of plants because it takes part in the synthesis of some amino acids such as tryptophan and tyrosine [1] that are fundamental for vegetal life.

Glyphosate is dramatically increasing in use in vast rural areas all around the world in association with the rise of genetically modified crop varieties specifically designed to be resistant to this herbicide. Unfortunately there is no known typical concentration of this agrotoxic in natural water bodies. The problem with this herbicide is the lack of control in very important agricultural regions in America and Asia while its use is controlled in most of the developed countries $[2,3]$. For drinking 
waters, USEPA and Health Canada have published Drinking Water Guidelines limiting Glyphosate concentrations to a maximum of $0.7 \mathrm{mg} / \mathrm{l}$ and $0.28 \mathrm{mg} / \mathrm{l}$ respectively $[4,5]$.

In some regions, light aircrafts are used to apply this herbicide by spraying it over the fields so the area of application is very poor selective and the product, in addition to settle on the crop to be treated, also precipitates on other non-desired areas affecting water [6,7], soil, plants and crops [8], wildlife and also directly and indirectly to humans [9].

Glyphosate has a decomposition period ranging from 7 to 60 days [10] but it can persist more than 174 days under certain environmental conditions [11] and, in addition, the toxicology of the product is under severe discussion. It is well known that, although it has not been shown to be carcinogenic yet, recent studies show its toxicity and its power as an endocrine disruptor [12]. Meanwhile, what happens with long term use of Glyphosate and the compounds derived from its degradation is still unknown.

On the other hand, the existing analytical techniques for detecting and quantifying Glyphosate and its derivatives in the physical environment (water and soil), animals or plants are complex and costly. In this sense, one of the most used methodologies is High Performance Liquid Chromatography-Mass Spectrometry (HPLC-MS) [13] although there are other methods like immunosorbent assays (ELISA) [13], Colorimetry [14], Caplillary Electrophoresys [15] and Electrochemiluminescence techniques, that make possible an effective analysis of Glyphosate [16]. However, these procedures are generally slow, expensive and they have to be performed in a laboratory, so the advance in the development of rapid, sensitive and "in situ" analytical procedures is a work of great interest [16].

In this regard, there are very important advances like those presented by Aquino et al [17] based on Electrochemistry and sensors made with different materials such as Silver and Platinum [18], Copper [19] or the very latest research with reactive enzymes [20] and double-layer hydroxides [21] which could be the basis for future "in situ" detection of Glyphosate. In this sense, it seems that Voltammetric / Amperometric techniques can be the basis for the development of these "in situ" measurement procedures. In fact, Voltammetry is the basis of the proposed procedure to predict Glyphosate concentration and a Voltammetric/Amperometric device called FraPlus has been designed and created to do Voltammetric/Amperometric measurements in a laboratory.

Finally, the goal of this paper is to present the results of the work developed to embed an ANN in a microcontroller in order to detect Glyphosate concentrations in a buffer dissolution by pulse voltammetry. To achieve this, several assays have been done with different concentrations of the herbicide and they have been analyzed using different metals (noble and not noble) as electrodes. The voltammetric data obtained by FraPlus have been used to create the mathematical models presented in this work.

\section{Materials and Methods}

\section{Chemical compounds}

In order to carry out the tests using pulse voltammetry, ten different dissolutions of Glyphosate $96 \%$ (purchased from Molekula) have been prepared, ranging from $5 \cdot 10^{-3} \mathrm{M}$ to $5 \cdot 10^{-5} \mathrm{M}$, using a buffer dissolution of sodium dihydrogen phosphate $0.1 \mathrm{M}$ (Sharlau, CAS: 7558-80-7) in distilled water, $\mathrm{pH}$ 6.7 achieved by adding a few drops of $\mathrm{NaOH} 1 \mathrm{M}$.

\section{Electrochemical measurement using pulse voltammetry}

The electrochemical measurement technique called pulse voltammetry has been used to apply an electric voltage to the utilized electrodes (working electrodes) when submerged in Glyphosate dissolutions, and then measure the electric current that circulates through the electrode. This is because the flow of the current through the dissolution is a function of the applied voltage and the chemical concentration in the dissolution.

The measurements have been made with a device developed by the Institute of Molecular Recognition and Technological Development (IDM) called FraPlusMini [22], which is capable of running tests for Potentiometry [23], Amperometry [23,24], Pulse Voltammetry and Cyclic Voltammetry [25], and has been successfully used in food [26-28], water analyses [29] and also in the determination of environmental parameters [30].

The system consists of a software application that runs on a PC and electronic equipment.

For Pulse Voltammetry, the equipment generates a sequence of up to 50 pulses with an amplitude that can be configured for each of the pulses in the range of $[-2 \mathrm{~V} ;+2 \mathrm{~V}]$. The width of the pulses can also be configured with a minimum value of $1 \mathrm{~ms}$ and a maximum value of $50 \mathrm{~ms}$.

The system includes a potentiostat that applies the voltage to the counter electrode of the electrochemical cell and measures the voltage at the reference electrode and the current at the 
working electrodes. The potentiostat permits measurements with up to 8 multiplexed working electrodes that can be automatically selected from the software application. As potentiostats are quite unstable systems, the design contains a stabilization circuit that can be configured according to the needs of each application. The current measurement circuit of the potentiostat disposes of 32 programmable current scales. Fig. 1 shows the block diagram of the designed system.

\section{[Insert Figure 1]}

The software application includes a section for pulse voltammetry. In this section, a sequence of tests for several working electrodes can be programmed. The application allows the setup of the pulse train parameters: number of pulses, amplitude of each pulse and width of the pulses. A different pulse pattern can be configured for each working electrode. The current scale and the stabilization level can also be established. Once the test has been configured the application sends this information to the electronic equipment. The application calculates 1000 points corresponding to the signal temporal evolution of the pulse train and sends this information (along with the rest of the data needed to carry out the test) to the electronic equipment through a USB port. Then, the electronic equipment generates and applies the pulse train to the electrochemical cell and samples the voltage and current signals. Finally the equipment sends these samples to the PC where the data is displayed and stored. This procedure is then repeated for the next working electrode of the sequence. The data of the complete sequence are stored in a file so that statistical studies can be applied.

The electronic equipment includes a 16-bit microcontroller (PIC24FJ256), a 12-bit Digital-to-Analog converter (DAC), two 12-bit Analog-to-Digital converters (ADC) and a potentiostat that incorporates a current measurement circuit, a working electrode multiplexing block and a stabilization circuit. Some analog signal conditioning circuits are used to adapt the signals that connect the potentiostat to the DAC and the ADC's.

The microcontroller receives the data sent by the PC. When all the data corresponding to a test is received, the microcontroller configures the current scale and the stabilization level of the potentiostat and selects the desired working electrode. Then it outputs the value corresponding to the temporal evolution of the signal to the DAC at a rate that fulfills the signal timing requirements. In the same loop the program of the microcontroller samples the signals corresponding to the voltage at the reference electrode and the current flowing through the selected working electrode. The collected data are sent to the PC where it is processed and stored.

\section{Electrochemical sensors (electrodes)}

In a preliminary approach, two electronic tongues have been designed, developed and used with two different sets of sensor materials (one with four noble metals: Ir, Rh, Pt and $\mathrm{Au}$; and another one with four non-noble metals: $\mathrm{Ag}, \mathrm{Cu}, \mathrm{Co}$ and $\mathrm{Ni}$ ) with a contact diameter of $1 \mathrm{~mm}$ for each metal. The selection of electrodes to our electronic tongues has been made according to our experience and papers published before [1][16][21][28]. After several preliminary tests we have decided to work with those more sensitive: Copper and Cobalt.

Once the valid electrodes have been identified, rotating disk electrodes have been built with $\mathrm{Cu}$ and Co with a contact diameter of $2 \mathrm{~mm}$ in order to confirm the effect of increasing the contact surface in the measurement sensibility and also the effect of the electrode rotation to avoid oxides to get fixed to the electrode surface. In this way, both surface increase and the turbulent flow created by the rotation help to increase the signal.

Besides, the use of a calomel (Radiometer Analytical, XR 100) reference electrode was needed.

Laboratory analyses.

Electrochemical measures by pulse voltammetry have been done to Glyphosate dissolved in buffer at ten different concentrations $\left(5 \cdot 10^{-3} \mathrm{~mol} \mathrm{dm}^{-3}, 2.5 \cdot 10^{-3} \mathrm{~mol} \mathrm{dm}^{-3}, 1.25 \cdot 10^{-3} \mathrm{~mol} \mathrm{dm}^{-3}, 0.625 \cdot 10^{-4} \mathrm{~mol}^{-3}\right.$ $\mathrm{dm}^{-3}, 5 \cdot 10^{-4} \mathrm{~mol} \mathrm{dm}^{-3}, 2.5 \cdot 10^{-4} \mathrm{~mol} \mathrm{dm}^{-3}, 1.25 \cdot 10^{-4} \mathrm{~mol} \mathrm{dm}^{-3}, 0.625 \cdot 10^{-4} \mathrm{~mol} \mathrm{dm}^{-3}, 5 \cdot 10^{-5} \mathrm{~mol} \mathrm{dm}^{-3}$, $2.5 \cdot 10^{-5} \mathrm{~mol} \mathrm{dm}^{-3}$ ) using the FraPlus device and a determinate pulse train. Preliminary assays have been done with eight different working electrodes made with noble and non-noble metals as described above. Next, specific analyses have been done with the most sensitive electrodes to these glyphosate concentrations by using rotating electrodes, due to their better response when compared with the static ones. Then, three different series of assays (A, B and C) have been carried out in order to have a statistically significant number of samples and check their repeatability.

After considering several pulse trains in preliminary assays and attending to previous papers in this area $[11,13,17]$, we have decided to use a pulse train that consists of nine different pulses both positive and negative in the voltage range of $[-500 \mathrm{mV}, 600 \mathrm{mV}]$ in order to capture the Glyphosate 
response to different voltages. These nine pulses last for $40 \mathrm{~ms}$ each and are the following ones: 0 $m V,-200 m V, 0 m V,+600 m V,-500 m V,-200 m V, 0 m V, 200 m V$ and $0 m V$.

After this, assays with rotating $\mathrm{Co}$ and $\mathrm{Cu}$ electrodes have been done. The voltammetric response database for the ten different Glyphosate concentrations to the nine pulses with Cobalt and Copper electrodes has been collected in a text file to be modeled by two different procedures.

Multivariate analysis of data

A data matrix has been built with the data collected for statistical calculations. The software Statgraphics ${ }^{\circledR}$ (StatPoint Technologies, Inc.) has been used for these analyses; Principal Component Analysis (PCA) and Partial Least Squares analyses (PLS) have been done with this software. PCA analyses were made in order to reduce the amount of input variables (usually dependent) in a lower number of independent variables (this allows their representation in two or three dimensions for the two or three principal components) and PLS analysis were made in order to obtain the factors of each component of the models and check their statistical validity.

Artificial Neural Network modeling

Neural networks can be applied in systems when it is necessary to develop a model (functional, classifier, predictive, etc.). They are ideal in applications with low density of data and information even though a large amount of input data. In addition, they present some interesting properties such as robustness to inconsistencies in input data, connections with mixed data and processing rules, highly parallel processing, self-organization and adaptability [31,32].

The procedure for working with artificial neural networks consists of two stages, a first stage of training of the network and a second stage for its verification. The training stage has been performed with some of the available measures. At this stage the network categories have been set out (in this case, ten different Glyphosate concentrations). The data from eight electrodes for each measurement have been applied as an initial input vector that was simplified later. With these data, the coefficients of the algorithm that configures the network have been calculated. In the verification stage, the data from new measures have been applied to the inputs, checking whether the output of the active network is correct or not.

As a part of further works in this research line, once validated the results, we will intend to create a portable system equipped with artificial intelligence that must be able to detect glyphosate by using the simplest and most economic procedure. Usually, portable systems use programmable components such as microcontrollers, DSP, FPGA, etc. In our case, we are planning to implement the algorithm in a microcontroller because of its ease of programming, comprehensive development tools and low production cost.

For implementation in portable equipment, the neural network algorithm must be transferable to a microcontroller which has a limited amount of memory. Thus, the neural network algorithm will require high accuracy and low memory requirements, as well as it must work fast and be able to work in realtime in order to be implemented in a microcontroller. In the end, not all neural network algorithms are able to reach all these requirements. In order to get it, two neural network algorithms have been used in this communication: Fuzzy ARTMAP and Multi-Layer Feed-Forward (MLFF).

\section{Results}

An important database has been obtained from the analyses done with Glyphosate dissolved in buffer at ten different concentrations by using the FraPlus device and a determinate pulse train. The results of applying this pulse train to the ten selected Glyphosate concentrations are 999 voltammetric measures corresponding to the voltammetric response of each dissolution to the applied voltages.

A comparison among the results for the eight different electrodes and their responses to the different Glyphosate concentrations has allowed us to decide working with $\mathrm{Co}$ and $\mathrm{Cu}$ electrodes. They have given the highest electrochemical response as they have been observed to have a clear tendency to be oxidized in the presence of this compound forming complex species. Due to their sensibility to Glyphosate concentrations, they have been selected as working electrodes.

After this, the assays with rotating $\mathrm{Co}$ and $\mathrm{Cu}$ electrodes have shown very interesting results. The results data base have been built including the 999 voltammetric data of 140 different assays with the rotating $\mathrm{Cu}$ electrode and 111 different assays with the rotating Co electrode. In this sense several tables as the one shown in Table 1 have been obtained for each analytical series (A, B and C) with the following voltammetric data: 
Next, an example of the global electrochemical responses obtained is shown in Fig. 2, corresponding to the averaged voltammetric results in series A for all pulses and the ten Glyphosate concentrations analyzed with the Cu electrode

$$
\text { [Insert Figure 2] }
$$

Finally, the voltammetric response database for the ten different Glyphosate concentrations to the nine pulses with Cobalt and Copper rotating electrodes has been collected in a text file to be modeled by two different procedures as shown in the next section.

\section{Mathematical modeling}

Two different mathematical modeling procedures have been used in this study in order to be able to predict Glyphosate concentrations in water samples by voltammetric techniques. The first one has been done by using PLS (Partial Leasts Squares). The second modeling procedure has been based on the theory of Artificial Neural Networks. The goal of these two different modeling processes is to be able to compare results and check the fit of these two different philosophies of modeling to the electrochemical response of Glyphosate dissolutions to voltammetric pulses.

4.1. $\quad$ Mathematical model by PLS

The idea of modeling by PLS is to have a flexible tool to obtain the mathematical expressions of the curves and be able to get a mathematical model based on our own design and not on a preconfigured one. In this sense, we have used Statgraphics ${ }^{\circledR}$ to model the response of Glyphosate obtained with the two most sensitive sensors identified in the laboratory assays: Cobalt and Copper.

a) For Cobalt electrode:

First of all we have calculated the areas determined by the curves of the nine pulses applied to the different Glyphosate concentrations assayed (111 assays have been done with the Co electrode). Next, the areas of the curves from the buffer have been subtracted to each one of the curves for each Glyphosate dissolutions, in order to use the buffer of each series as a reference to compare results.

After this, we have developed a method to determine the Glyphosate concentration based on considering the areas for each pulse as independent variables. The idea was to create a model in the following form:

[ Glypho $(\mathrm{mg} / \mathrm{l})]=\mathrm{a}+\mathrm{b} \cdot \mathrm{P} 1+\mathrm{c} \cdot \mathrm{P} 2+\mathrm{d} \cdot \mathrm{P} 3 \ldots . . \mathrm{i} \cdot \mathrm{P} 9$

In this expression, [Glypho $(\mathrm{mg} / \mathrm{l})$ ] is the predicted value of Glyphosate concentration and $P_{i}$ is the value of the area corresponding to each one of the nine pulses used. Lastly, letters, $a, b, c \ldots$ to $i$, are coefficients given by the PLS analysis.

In this specific case, we have chosen only three of the nine pulses applied in the assays due to the fact that this is what a preliminary Principal Components Analysis (PCA) of the results suggests. In fact, the output of the applied PLS analysis showed the result of fitting models for 1 dependent variable based on 3 predictor variables. To fit the model, 2 components were extracted. In the end, the P-Value of the model is 0.0 and the value of $R$ is 0.9793 so the proposed model is statistically valid (Table 2).

\section{[Insert table 2]}

As shown in table 2, the selected areas were the ones for pulses P2, P4 and P8. In this way, the mathematical model to predict Glyphosate concentrations based on the selected areas of the voltammetric responses to the pulse train previously described is as shown: 
The mathematical model validation has been made by "leave one out".

b) For Copper electrode:

We have proceeded in the same way with the results of the electrochemical analyses with the $\mathrm{Cu}$ electrode. In this sense, the mathematical model was in the same form than the one shown before but considering four predictor variables as suggested by a previous PCA analysis. In order to fit the model, three components were extracted as shown in table 2 . Then, the mathematical model is in the following form:

\section{[Insert Figure 3]}

$[$ Glypho $(\mathrm{mg} / \mathrm{l})]=2.58091+8.51427 \cdot \mathrm{E}^{-3} \cdot \mathrm{P} 3+1.6872 \cdot \mathrm{E}^{-3} \cdot \mathrm{P} 4-4.91523 \cdot \mathrm{E}^{-3} \cdot \mathrm{P} 8+0.103068 \cdot \mathrm{P} 9$

In this case, P-value was 0.0 and R-Squared was 0.9955 for the predicted versus observed values (Fig. 3) so the mathematical model is considered statistically valid. As done with the model for Co electrode, the validation of the model was made by "leave one out".

4.2. Mathematical model by neural networks.

In order to develop the model, we have used a very extended and versatile artificial neural network: feed-forward multi-layer perceptron neural network (FF-MLP). The network is a two layer feedforward network with sigmoid hidden neurons and linear outputs neurons. The first layer has a connection from the network input; in this specific case, we have four inputs for Copper corresponding to pulses $\mathrm{P} 3, \mathrm{P} 4, \mathrm{P} 8$ and $\mathrm{P} 9$ and three inputs for Cobalt corresponding to pulses P2, P4 y P8. For each one of these inputs we have 127 data corresponding to the different molar concentrations used in the assays. The number of the hidden nodes selected is 10 . The activation function used for the hidden neurons is the type Tansig (Hyperbolic tangent sigmoid transfer function). Purelin (linear) function is used for the output node.

\section{a) For Copper electrode:}

Random data divisions have been used by Matlab $®$ in order to select the samples for the training. $70 \%$ of the samples have been used training data, $15 \%$ as validation data and the remaining $15 \%$ has been used as test data. After the training, two weight matrices have been obtained: a 20x4 hidden weight matrix and in the other hand, a $4 \times 1$ output weight matrix. In addition, two bias matrices were also obtained: a $4 \times 1$ hidden bias matrix and a 1x1 output bias matrix. A maximum and minimum of the input data have been also obtained.

Values of MSE (Mean Squared Error) and R (Regression Coefficient) are shown in Table 3. Fig. 4 shows $\mathrm{R}$ values for every sample (training, validation and test) as well as for all sample groups.

[Insert table 3]

Despite of getting a good value of $R=0.999$ in the regression line, there is a high dispersion at low concentration values. When representing measured values versus those predicted by the neural network, it can be seen that there is a very good value at high and medium concentrations $(R=$ 0.9504 for medium concentration and $\mathrm{R}=0.9992$ for high concentration) but the relationship is bad at low concentrations. 
Since the results at low concentrations are not the desired ones, we decided to use a previous phase for classifying the data before the modeling phase with the idea of getting a better $\mathrm{R}$ value. In our case, we classified the data into three different classes: Class 1 (Low concentrations), Class 2 (medium concentrations) and Class 3 (High concentrations). This classification was performed by using a neural network Fuzzy ARTMAP type as explained in section c).

[Insert Figure 4]

b) For Cobalt electrode:

In the specific case of using a Cobalt electrode we have realized that the results are not as good as those obtained by using the Copper one so we have decided to develop the Artificial Neural Network considering only the Copper electrode.

c) For Copper electrode with previous classification:

As cited before, a previous classification of the input data has been done. The idea is to improve the model created by the FFMLP. Next, the data classification has been made with the other neural network (Fuzzy ARTMAP)[33] [34] that is based on the Adaptative Resonance Theory [35] and is able to learn in an unsupervised mode with a fast learning rate [36].

As explained before, in order to train the network, a classification of the obtained data into three different classes has been done (low concentration (1), medium concentration (2) and high concentration (3)) as shown in Table 4.

\section{[Insert table 4]}

In each selected class 38 data have been used so the study is developed with 114 input data. A part of these data has been used to do the training (90 data) and another part to do de validation (24).

In order to reach a compromise between a high hit rate and a minimum mapfield, the $\rho$ values have been changed in the range of $[0.3-0.9]$ and $\beta$ values have also changed in the range of [0.4 - 1$]$. The goal of getting a minimum mapfield is based on the idea of reducing the use of the microcontroller memory when this model became developed into a portable system.

A small sized mapfield has been obtained with values in the range of [0.3-0.6] and in the range of [0.6-1] with a hit rate of $87.5 \%$. Maximum hit rate is obtained for $\rho=0.9$ and for $\beta=0.6,0.7$ and 1 getting a mapfield of 18,21 and 17 respectively. Minimum mapfield is obtained with $\rho=0.9$ and $\beta$ $=1$. With this, we obtain a weight matrix of $17 \times 8$.

\section{[Insert Table 5]}

Fig. 5 shows the confusion matrix. In the confusion matrix, the diagonal cells show the number of residue positions that were correctly classified for each structural class. The off-diagonal cells show the number of residue positions that were misclassified. The blue cell shows the total percentage of correctly predicted residues (top number) and the total percentage of incorrectly predicted residues (bottom number).

\section{[Insert Figure 5]}

After having classified the input data with the Fuzzy ARTMAT network, a FFMLP network has been developed in order to predict the concentration for each of the 3 classes, so three independent networks were obtained for low, medium and high concentrations.

For class 1, a FFMLP architecture with 4 inputs (for pulses P3, P4, P8 and P9) has been used. We have also used 16 neurons for hidden layer and one neuron for the output layer. This architecture leads to a $16 \times 4$ weight matrix for the hidden layer and a $16 \times 1$ matrix for the bias. In the end, the output weights form a matrix of $1 \times 16$ and $1 \times 1$ bias. The obtained value for $R$ is 0.932 , it is notably 
better than the one obtained with no preliminary data classification $(R=0.0632)$ and it confirms that a previous classification gives better results.

For classes 2 and 3 the best result was obtained using a network with 10 neurons in the hidden layer

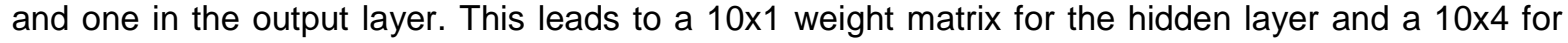
the bias. Finally, the output weights form a matrix of $1 \times 10$ and $1 \times 1$ bias. In this case, Regression Coefficients are 0.9862 and 0.9992 respectively for Classes 2 and 3. In this case the Regression Coefficient for Class 2 is better than the one corresponding to the previous no classification case (0.9504) while Class 3 has the same $R$ value. These $R$ values mean that the adjustment of the model is very good and Glyphosate concentration in water samples can be predicted in the studied concentration range.

In the end, results for Class 1 (low Glyphosate concentrations) can be further improved if data are preprocessed trying to eliminate outliers. In order to do this, a univariate outlier detection method has been applied as explained by Ben-Gal\&Walfish $[37,38]$. This method consists of three steps. First, the outlier detection method is applied to the inputs. Next, the classification is performed and, finally, settings are done. In our case, when outliers elimination is applied, a R value of 0.9583 is obtained for Class 1 and this is better than the Regression Coefficient obtained without applying this method $(R=0.815)$. Finally, we have proceeded in the same way with the overall Glyphosate concentration data to get a global Regression Coefficient for our prediction model $(R=0.9998)$. This ANN based mathematical model is the best choice to predict Glyphosate concentrations in buffer dissolutions and so we suggest this model to be applied to the detection of this pesticide in natural water bodies.

[Insert Figure 6]

\section{Conclusions}

It's well known that Glyphosate is the world's most used herbicide and that there are ecological problems and human affections due to its use. In addition, there are no easy, fast and economic techniques for its detection neither in a laboratory nor "in situ". Voltammetric/Amperometric techniques with metal electrodes seem to be giving promissory results in the detection and quantification of organic compounds in water.

We have developed a Voltammetry device called FraPlus that allows us to detect glyphosate concentrations in a buffer. Assays with different Glyphosate concentrations have given a database to build a mathematical model by both: statistical methods (PLS) and ANN procedures.

In the end, ANN is the best choice to create a mathematical model as it gives the best statistical results specially when a previous classification of the samples is done in three different Classes: low (class 1 ), medium (class 2), and high concentrations (class 3 ). In this specific case, Regression Coefficient value and P-Value are the best of all the studied models $(R=0.9998$ and $P$-value $=0.0)$ so we recommend this procedure for further development of mathematical models to predict Glyphosate concentrations in real water samples.

\section{Acknowledgements}

Authors acknowledge financial support from the Universitat Politècnica de Valencia and its Centre de Cooperació al Desenvolupament (Programa ADSIDEO-COOPERACIÓ 2010) for the research fellowship and support given by UNIJUI University \& Staff during the stage of Prof. Laguarda in ljuí (RS-Brazil). We also thank MICINN (MAT2009-14564-C04-02) and GVA (PPC/2011/019) for their respective research fellowships.

\section{References:}

[1] E.V. Sierra, M.A. Méndez, V.M. Sarria, M.T. Cortés. Electrooxidation of glyphosate on nickel and Copper electrodes. Quimica Nova 31 (2008). 
[2] Ministerio de la Presidencia. Orden PRE/876/2007 de 30 de Marzo por la que se modifican los anexos II de los Reales Decretos 569/1990 de 27 de abril y 280/1994 de 18 de febrero, por los que se establecen los límites máximos de residuos plaguicidas y su control en determinados productos de origen animal y vegetal, respectivamente. Boletín Oficial del Estado 83 (2007) pp 37.

[3] Comisión de las Comunidades Europeas. DIRECTIVA 2006/60/CE DE LA COMISION de 7 de julio de 2006 que modifica los anexos de la Directiva 90/642/CEE del Consejo por lo que especta a los limites maximos de residuos de trifloxistrobin, tiabendazol, abamectina, benomilo, carbendazima, tiofanato-metil, miclobutanil, glifosato, trimetilsulfonio, fenpropimorf y clormequat. Diario Oficial de la Unión europea 206( 2006) pp 11.

[4] USEPA. 2012 Edition of the Drinking Water Standards and Health Advisories. United States Environmental Protection Agency. (2012). (http://www.epa.gov/).

[5] Glyphosate. Guidelines For Canadian Drinking Water Quality - Supporting Documents - Glyphosate. Health Canada. (1995). (www.hc-sc.gc.ca)

[6] C. Cox. Glyphosate (Roundup). J. Pest. Reform. 18 (1998) 3-17.

[7] F. Nirascou. Les pesticides dans les eaux. Dossier 5 IFEN (2006) pp36.

[8] J.W. Sawchuk, R.C.V Van Acker, L.F. Friesen. Influence of a Range of Dosages of MCPA, Glyphosate, and Thifensulfuron:Tribenuron (2:1) on Conventional Canola (Brassica napus) and White Bean(Phaseolus vulgaris) Growth and Yield. Weed Technol. 20 (2006) 184-197.

[9] V. Contardo-Jara, E. Klingelmann, C. Wiegand, Bioaccumulation of glyphosate and its formulation Roundup Ultra in Lumbriculus variegatus and their effects on biotransformation and antioxidant enzymes. Environ. Pollut. 157 (2009) 57-63.

[10] C. Accinelli, C. Screpanti, A. Vicari, P. Catizone. Influence of insecticidal toxins from Bacillus thuringiensissubsp. kurstaki on the degradation of glyphosate and glufosinate-ammonium in soil samples. Agr. Ecosyst \& Environ. 103 Issue 3,(2004) $497-507$.

[11] Hoja de manejo seguro. ESTELAR 480 SL. Dow Chemical. 1999.

[12] C. Gasnier, C. Dumont, N. Benachour, E. Clair, M-C. Chagnon, G-E. Séralini. Glyphosate-based herbicides are toxic and endocrine disruptors in human cell lines. Toxicology 262 (2009) 184-191.

[13] F. Sánchez-Bayo, R.V: Hyne, K.L. Desseille. An amperometric method for the detection of amitrole, glyphosate and its aminomethyl-phosphonic acid metabolite in environmental waters using passive samplers. Anal. Chim. Acta 675 (2010) 125 131.

[14] C.F.B. Coutinho, L.F.M. Coutinho, L.H. Mazo, S.L. Nixdorf, C.A.P. Camara. Rapid and direct determination of glyphosate and aminomethyl phosphonic acid in wáter using anion-exchange chromatography with coloumetric detection. J. Chromatogr. A 1208 (2008), $246-249$.

[15] H.Y. Chiu, Z.Y. Lin, H. Tu, Ch. Whang. Analysis of glyphosate and aminomethylphosphonic acid by capillary electrophoresis with electrochemiluminiscence detection. J. Chromatogr. A 1177 (2008) 185 - 198.

[16] J. Jin, F. Takahashi, T. Kaneko, Toshio Nakamura. Characterization of electrochimiluminiscence of tris (2,2'bipiridine)ruthenium (II) with glyphosate as coreactant in aqueous solution. Electrochim. Acta 55 (2010) 5532 - 5537.

[17] S. Aquino Neto, A.R. de Andrade. ELectrooxidation of glyphosate herbicide at different DSA compositions: pH, concentration and supporting electrolyte effect. Electrochim. Acta 54 (2009), 2039 - 2045.

[18] M.A. Méndez, M.F. Suárez, M.T. Cortés, V.M. Sarria. ELectrochemical properties and electro-aggregation of silvercarbonate sol on polycrystalline platinum electroda and its alactrocatalytic activity towards glyphosate oxidation. Electrochem. Commun 9 (2007) $2585-2590$.

[19] C.F.B. Coutinho, M.O. Silva, M.L. Calegaro, S.A.S. Mahcado, L.H. Mazo. Investigation of Copper dissolution inthe presence of glyphosate using hydrodynamic voltammetry and chronoamperometry. Solid State Ionics 178 (2007) 161 -164.

[20] E.A. Songa, O.A. Arotiva, J.H.O. Owino, N. Jahed, P.G.L. Baker, E.I. Iwuoha. Electrochemical detection of glyphosate herbicide using horseradish peroxidase immobilized on sulfonated polymer matrix. Bioelectrochemistry 75 (2009) 117 - 123.

[21] A, Khenifi, Z. Derriche, C. Forano, V. Prevot, C. Mousty, E. Scavetta, B. Ballarin, L. Guadagnini, D. Tonelli. Glyphosate and glufosinate detection at electrogenerated NiAl-LDH thin films. Anal. Chim. Acta, 654, 2 (2009) 97 - 102.

[22] R. Masot, M. Alcañiz, A. Fuentes, F.C. Schmidt, L. Gil, D. Baigts, R. Martínez-Máñez, J. Soto, J.M. Barat. Design of a low-cost non-destructive system for punctual measurements of salt levels in food products using impedance spectroscopy. Sens. Actuators A 158 (2010) 217-223.

[23] J.M. Barat, L. Gil, E. García-Breijo, M-C. Aristoy, F. Toldrá, R. Martínez-Máñez, J. Soto. Freshness monitoring of sea bream (Sparus aurata) with a potentiometric sensor. Food Chem. 108 (2008) 681-688. 
[24] I. Campos, R. Masot, M. Alcañiz, L. Gil, J.L. Vivancos, E. García-Breijo, R. H. Labrador, J. M. Barat, R. Martínez-Máñez, J. Soto. Accurate concentration determination of anions nitrate, nitrite and chloride in minced meat using a voltammetric electronic tongue. Sens. Actuators B 149 (2010) 71-78.

[25] E. García-Breijo, J.M. Barat, O.L. Torres, R. Grau, J. Ibáñez, M. Alcañiz, R. Masot, R. Fraile, L. Gil. Development of a puncture electronic device for electrical conductivity measurements throughout meat salting. Sens. Actuators A 148 (2008), 63-67.

[26] L. Gil, J.M. Barat, E. Garcia-Breijo, J. Ibañez, R. Martínez-Máñez, J. Soto, E. Llobet, J. Brezemes, M-C. Aristoy, F. Toldrá. Fish freshness analysis using metallic potentiometric electrodes. Sens. Actuators B 131 (2008) 362-370.

[27] R.H. Labrador, R. Masot, M. Alcañiz, D. Baigts, R. Martínez-Máñez, E. García-Breijo, L. Gil, J. Soto, J.M. Barat. Prediction of $\mathrm{NaCl}$, nitrate and nitrite contents in minced meat by using a voltammetric electronic tongue and an impedimetric sensor. Food Chem 122 (2010) 864-870.

[28] L. Gil, J.M. Barat, D. Baigts, R. Martínez-Máñez, E. García-Breijo, M-C. Aristoy, F. Toldrá, J. Soto, E. Llobet. Monitoring of physical-chemical and microbiological changes in fresh pork meat under cold storage by means of a potentiometric electronic tongue. Food Chem. 126 (2011) 1261-1268.

[29] E. Garcia-Breijo, J. Atkinson, L. Gil-Sanchez, R. Masot, J. Ibañez, J. Garrigues, M. Glanc, N. Laguarda-Miro, C. Olguin. A comparison study of pattern recognition algorithms implemented on a microcontroller for use in an electronic tongue for monitoring drinking waters. Sens. Actuators A: Phys. (2011), doi:10.1016/j.sna.2011.09.039.

[30] J. Ibáñez Civera, E. Garcia Breijo, N. Laguarda Miró, L. Gil Sánchez, J. Garrigues Baixauli, I. Romero Gil, R. Masot Peris, M. Alcañiz Fillol. Artificial neural network onto eight bit microcontroller for Secchi depth calculation. Sens. Actuators B 156 (2011) 132-139.

[31] B. Martin del Rio. Redes Neuronales y Sistemas Borrosos. Ed. Rama, Madrid, 2006.

[32] P. Neelamegam, A. Rajendran, Neural network based density measurement. Bulg. J. Phys. 31 (2004) 163-169.

[33] C.M. Bishop, Neural Networks for Pattern Recognition. Oxford Univ. Press, New York, 1995.

[34] N. Medrano-Marqués, B. Martin-del-Brío, Implementing neural networks onto standard low-cost microcontroller for sensor signal processing, in: Emerging Technologies and Factory Automation, 2005. ETFA 2005. 10th IEEE Conference, September, 2005, pp. 972-978.

[35] G.A. Carpenter, S. Gossberg, ART2: self-organization of stablecategory recognition codes for analog input patterns, Appl. Optics 26 (1987) 4919-4930. Page 24 of 51 Accepted Manuscript 24

[36] G.A. Carpenter, S. Gossberg, N. Markuzon, J. Reynolds, D. Rosen, Fuzzy Artmap: A Neural Network architecture for incremental supervised learning of analog multidimensional maps, IEEE Transactions on Neural Networks 3 (1992) 698-713.

[37] I. Ben-Gal, Outlier detection, In: O. Maimon \& L. Rockach (Eds.) Data Mining and Knowledge Discovery Handbook: A Complete Guide for Practitioners and Researchers," Kluwer Academic Publishers, Tel-Aviv University, Israel, 2005.

[38] S. Walfish, A Review of Statistical Outlier Methods. PharmTech. 2 Nov (2006) 1-5. 
Vitae:

Nicolás Laguarda Miró was graduated in Environmental Sciences from the Universidad Politécnica de Valencia (UPV) in 2000. He received his PhD from the same university in 2005. Nicolás is nowadays a member of the Institute of Molecular Recognition and Technological Development (IDM) as well as a teacher of environmental subjects in the ETSID (Escuela Técnica Superior de Ingeniería del Diseño) at UPV. His main areas of interest are the environmental quality control, environmental impacts assessment and technology applications to these areas.

Francesca Werner Ferreira was graduated in Biology Sciences in 1983 and obtained her postgraduate in Zootechnics in 1989. She is a Ph. Doctor in Zootechics at the Universidade Federal de Santa Maria, Brazil, in 2008. Nowadays she is na assistang professor at the Universidade Regional do Noroeste do Estado do Rio Grande do Sul-UNIJUI (Brazil). Her main área of interest is Zoology especially ictiofauna diagnostic and monitoring, Compared Animal Physiology, Pisciculture of native and Environmental Impact Assessment.

Eduardo García Breijo was graduated in electronic engineering from the Universitat de València (Spain) in 1997, and received his PhD in 2004 from the Universidad Politécnica de Valencia (UPV). $\mathrm{He}$ is an assistant professor of Electronic Technologic of UPV. He is a member of the Institute of Molecular Recognition and Technological Development (IDM). His main areas of interest are the development of multisensors in thick-film technology.

Javier Ibáñez Civera is Matrisse in Power Electronic and Control from the Universite Pierre et Marie Curie (Paris VI) in 1994 and received his PhD in 2009 from the Universidad Politécnica de Valencia (UPV). He is assistant professor of Electronic Technology in the Electronic Engineering Department of the Universidad Politécnica de Valencia (UPV). He is a member of the Institute of Molecular Recognition and Technological Development (IDM). His main areas of interest are water organic contamination devices.

Luis Gil Sánchez was graduated in electronic engineering from the Universitat de Valencia (Spain) in 1998, and received his PhD in 2007 from the Universidad Politécnica de Valencia (UPV). He is assistant professor of electronic technology in the Electronic Engineering Department at UPV. He is a member of the Institute of Molecular Recognition and Technological Development (IDM). His main areas of interest are the chemical sensors, instrumentation systems and pattern recognition for electronic tongues.

José Garrigues Baixauli was graduated in Industrial Engineering from the Universidad Politécnica de Valencia in 1983. Since 1990, he is a member of the staff at the Electronic Department and he is also a member of the Institute of Molecular Recognition and Technological Development (IDM) in this University. His main areas of interest are Artificial Neural Networks applications (ANN) by microcontrollers. Nowadays he is developing his Thesis in these applications. 